\title{
Características clínico-epidemiológicas de pacientes con múltiples cánceres primarios en un hospital de Chiclayo
}

\author{
Dalia Carrasco-Ramos 1,a, Kenny Chonlon-Murillo 1,a, Jorge Fernández 1,a, Sebastian Iglesias-Osores ${ }^{2, b}$, \\ Alain Monsalve-Mera ${ }^{1, b}$
}

\section{RESUMEN}

Objetivos. Describir las características clínico-epidemiológicas de los pacientes con múltiples cánceres primarios en un hospital de Chiclayo, Perú durante los años 2009-2015. Material y métodos. Estudio descriptivo transversal. Se identificaron a los pacientes que padecían de más de un cáncer primario en el registro hospitalario de cáncer del Hospital Nacional Almanzor Aguinaga Asenjo, Chiclayo, Perú. Usando una ficha de recolección de datos se recolectó información acerca de la edad, el estadio del cáncer, fecha de diagnóstico, tipo de tumor, presencia de factores de riesgo para el desarrollo de más de un tipo de cáncer, entre otros. Resultados. Durante los años 2009 al 2015 se diagnosticaron 8075 casos de cáncer, de los cuales 89 pacientes $(1,1 \%)$ presentaron múltiples cánceres primarios. De éstos, 32 pacientes fueron incluidos el estudio. Los múltiples cánceres primarios fueron más frecuentes en el sexo femenino. Los factores de riesgo son los hábitos nocivos (cuatro pacientes) y el tratamiento de la neoplasia previa (17 pacientes). La edad promedio al diagnóstico del primer cáncer primario fue de 66 años, y el promedio de edad en mujeres (60 años) fue menor que en varones (72 años). Conclusiones. El primer cáncer primario más frecuente fue el de mama, y la asociación más frecuente el de piel y próstata. El tipo de cáncer más frecuente fue el cáncer metacrónico tanto en varones como en mujeres. Ningún varón presentó cáncer sincrónico.

Palabras clave: Neoplasias, Tumores Malignos, neoplasias primarias (Fuente: DeCS-BIREME).

\section{Clinical-epidemiological characteristics of patients with multiple primary cancer in a Chiclayo's hospital}

\section{ABSTRACT}

Objectives. Describe the clinical-epidemiological characteristics of patients with multiple primary cancers in a hospital in Chiclayo, Peru during the years 2009-2015. Material and methods. Cross-sectional descriptive study. Patients with more than one primary cancer were identified in the hospital cancer registry of the Hospital Nacional Almanzor Aguinaga Asenjo, Chiclayo, Peru. Using a data collection form, information was collected about age, cancer stage, date of diagnosis, type of tumor, presence of risk factors for the development of more than one type of cancer, among others. Results. During the years 2009 to 2015,8 075 cases of cancer were diagnosed, of which 89 patients $(1.1 \%)$ had multiple primary cancers. Of these, 32 patients were included in the study. Multiple primary cancers were more frequent in the female sex. Risk factors are harmful habits (four patients) and treatment of previous malignancy (17 patients). The average age at diagnosis of the first primary cancer was 66 years, and the average age in women (60 years) was lower than in men (72 years). Conclusions. The first most frequent primary cancer was breast cancer, and the most frequent association was skin and prostate cancer. The most frequent type of cancer was metachronous cancer in both men and women. No male developed synchronous cancer.

Keywords: Neoplasms, Malignant Tumors, Primary Neoplasms (Source: MeSH-NLM).

\footnotetext{
Facultad de Medicina, Universidad Católica Santo Toribio de Mogrovejo. Chiclayo, Perú

${ }^{2}$ Hospital Regional Lambayeque. Chiclayo, Perú

${ }^{a}$ Médico Cirujano

${ }^{b}$ Biólogo 


\section{INTRODUCCIÓN}

En los últimos años la carga de enfermedad por cáncer se ha incrementado, lo que constituye un problema de salud pública a nivel mundial (1). En América se registraron 2,8 millones de casos nuevos y 1,3 millones de muertes por cáncer en 2012. Aproximadamente un $47 \%$ de las muertes por cáncer en las américas en el 2012 se produjeron en América Latina y el Caribe (2). Según el análisis de la situación del cáncer en el Perú (ASIS) en el período 2006-2011 se notificó 109914 casos, con un promedio 18319 casos nuevos por año, así el número de casos incidentes varió entre 16671 (año 2006) y 19461 (año 2011). Los cánceres que a nivel nacional presentaron un mayor promedio de casos por año fueron los de cérvix, estómago, mama, piel y próstata (1). El número de años de vida saludables perdidos por enfermedad (AVISA) por cáncer en el Perú durante el 2008 fue de 418960 años el cual representa el $8 \%$ de la carga de enfermedad nacional y constituye la cuarta causa más importante de carga de enfermedad con una pérdida de 15,8 años por cada 1000 habitantes ${ }^{(1)}$.

El concepto de múltiples cánceres primarios (MCP) fue empleado por primera vez por Bilroth en $1889{ }^{(3)}$, pero no fue hasta 1932 donde Warren y Gates establecieron los criterios anatomo clínicos para su diagnóstico (4). Desde entonces se ha venido reportando múltiples casos de neoplasias malignas primarias dobles, triples hasta incluso quíntuples ${ }^{(5,6)}$. Los MCP se definen como la presencia de dos o más neoplasias primarias en diferentes órganos o de diferentes tipos celulares en el mismo órgano basado en las normas de la International Association of Cancer Registries (IACR) (7). Estos se clasifican según el intervalo de tiempo que ocurre entre las neoplasias, dividiéndose en: neoplasia sincrónica, aquella que se diagnostica al mismo tiempo o dentro del intervalo de seis meses del diagnóstico de la primera neoplasia o llamada neoplasia índice; y neoplasia metacrónica, a aquella que se diagnostica después de más de 6 meses de la primera neoplasia (8).

La frecuencia de MCP también ha ido en aumento; sin embargo, los estudios acerca de su frecuencia, características clínico-epidemiológicas y factores asociados son escasos. Los estudios encontrados al respecto, por lo general, están enfocados a demostrar los MCP a partir de una neoplasia en un órgano específico y no se ha encontrado estudios que mencionen los MCP de manera global, independientemente del cual sea el órgano primario de la neoplasia (9).

La prevalencia de MCP se encuentra entre $0,73 \%$ y $11,7 \%$ (9). Así mismo, los pacientes que han desarrollado un tumor primario presentan mayor riesgo de desarrollar un segundo tumor primario ${ }^{(4-13-14)}$, se encontró un riesgo de $8,4 \%$ para hombres y de $8,7 \%$ para mujeres en el año $2001{ }^{(12)}$. El objetivo de este trabajo fue describir las características clínico-epidemiológicas de los pacientes con múltiples cánceres primarios en un hospital de Chiclayo, Perú durante los años 2009-2015.

\section{MÉTODOS}

\section{Diseño del estudio y población}

Se realizó un estudio observacional de tipo transversal descriptivo a partir de la información contenida en el registro hospitalario de cáncer del Hospital Nacional Almanzor Aguinaga Asenjo (HNAAA) del distrito de Chiclayo-Perú, el cual pertenece al Seguro Social de Salud (EsSalud). Dicho registro se encuentra en el área de epidemiología. Se realizó la búsqueda de los pacientes de los servicios de oncología, que cuentan con más de un cáncer primario consignado en dicho registro de la historia clínica, desde el año 2009 al 2015. Se excluyó a los pacientes que cuente solo con un tipo de cáncer en su diagnóstico. No hubo un universo en la muestra y la recolección fue censal.

\section{Técnicas e instrumentos de recolección de datos}

Se realizó una ficha de recolección de datos donde se registraron los datos obtenidos en las historias clínicas vigentes de los pacientes portadores de dos o más cánceres primarios documentados histológicamente, con mismo sitio primario y diferente grupo histológico o en diferente sitio primario y con diferente grupo histológico o en diferente sitio primario y mismo grupo histológico que no sea invasión. Los pacientes sin confirmación histopatológica concluyente o clara y los pacientes en los que el segundo tumor maligno es sospechoso de ser metástasis o invasión del primario fueron excluidos del estudio.

La recolección fue de enero a marzo y se consideró: edad, sexo, procedencia, ocupación, fallecido o no, antecedentes patológicos personales, presencia de factores de riesgo para cáncer y características de cada cáncer que presente el paciente (fecha de diagnóstico, órgano primario, tipo de tumor, grado de diferenciación, edad al diagnóstico, estadio clínico (TNM), tipo de tratamiento, remisión del cáncer y fecha de admisión en el hospital).

\section{Descripción de las variables de estudio}

Los pacientes se evaluaron desde el punto de vista clínico, incluyendo: edad, sexo, fallecido o no fallecido, ocupación y procedencia. Se documentaron los antecedentes patológicos personales: hipertensión arterial, diabetes mellitus, hipertiroidismo, hipotiroidismo, hepatitis $B$, Virus Papiloma Humano (VPH), obesidad y otros. Además, se obtuvo la presencia o no de factores de riesgo de cáncer: consumo de tabaco, consumo de alcohol, radioterapia, quimioterapia, sedentarismo, tratamiento hormonal, genético/hereditario y dieta.

Los Tipos de neoplasia primaria múltiple se clasificaron como: "sincrónico" para aquellas neoplasias que se diag- 
nosticaron al mismo tiempo o dentro del intervalo de 6 meses del diagnóstico de la primera neoplasia y "metacrónico" a aquellos cánceres que se diagnosticaron después de más de 6 meses de la primera neoplasia.

Además, se documentó el tipo de tratamiento que recibieron para la neoplasia presentada: tratamiento quirúrgico paliativo, tratamiento quirúrgico curativo, quimioterapia, radioterapia, tratamiento paliativo/ sintomático, medicina nuclear, terapia biológica, hormonoterapia e inmunoterapia.

\section{Análisis estadístico}

Para el análisis de los resultados se empleó el programa Microsoft Excel. Para las variables cuantitativas se utilizaron medidas de tendencia central y de dispersión, mientras que para las variables cualitativas se utilizaron frecuencias y porcentajes presentados en diagramas, tablas y/o gráficos.

\section{Consideraciones éticas}

El proyecto del estudio fue revisado y aprobado por el Comité de Ética en Investigación de la Facultad de Medicina de la Universidad Católica Santo Toribio de Mogrovejo, así como por el Comité Institucional de Ética en Investigación del Hospital Nacional Almanzor Aguinaga Asenjo. Este último tras aprobar el proyecto autorizó el acceso a la información de la base de datos/historias clínicas del registro de cáncer del Área de epidemiología. Se utilizaron códigos para identificar a los participantes. La base de datos original fue registrada en una hoja de Microsoft Excel, a la cual solo tuvieron acceso los investigadores.

\section{RESULTADOS}

Durante los años 2009 al 2015 se diagnosticaron en el HNAAA 8075 casos de cáncer, de los cuales 89 pacientes $(1,1 \%)$ presentaron múltiple cáncer primario (MCP). De éstos, 32 pacientes fueron considerados para el estudio. (Figura 1). Cabe mencionar que no se tuvo accesibilidad a 57 historias clínicas debido a la inadecuada organización de historias clínicas en el hospital donde se realizó el estudio.

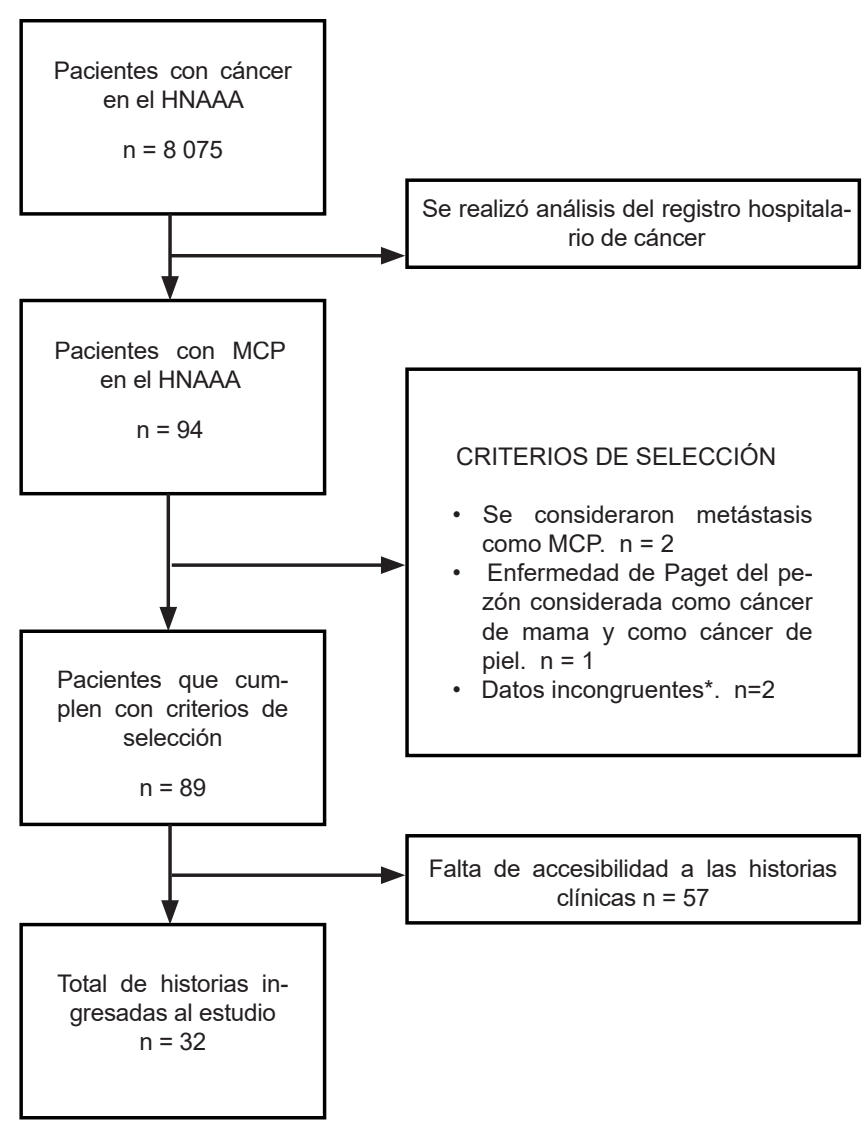

*Neoplasias benignas consideradas como malignas

Figura 1: Diagrama de flujo de pacientes con múltiples cánceres primarios.

Se encontró que los múltiples cánceres primarios fueron más frecuentes en el sexo femenino (Tabla 1). Los casos registrados fueron más frecuentes en el distrito de Chiclayo. La Ocupación más frecuente fue ser ama de casa. La frecuencia de factores de riesgo fue del $16,9 \%$, siendo el factor de riesgo más frecuente el tratamiento de neoplasia $6,3 \%$. 
Tabla 1. Características clínico- epidemiológicas de pacientes con múltiples cánceres primario en un hospital de Chiclayo, Perú durante los años 2009-2015 ( $\mathrm{N}=32)$.

\begin{tabular}{|c|c|c|}
\hline Variables & $\mathrm{N}$ & $\%$ \\
\hline \multicolumn{3}{|l|}{ Procedencia } \\
\hline Chiclayo & 22 & 68,8 \\
\hline Otros distritos & 10 & 31,3 \\
\hline \multicolumn{3}{|l|}{ Antecedentes } \\
\hline Hipertensión & 21 & 65,6 \\
\hline Diabetes & 7 & 21,9 \\
\hline Obesidad & 2 & 6,3 \\
\hline Hipotiroidismo & 2 & 6,3 \\
\hline \multicolumn{3}{|l|}{ Hábitos nocivos } \\
\hline Consumo tabaco* & 2 & 6,3 \\
\hline $\begin{array}{l}\text { Consumo de } \\
\text { alcohol* }\end{array}$ & 2 & 6,3 \\
\hline Sedentarismo & 2 & 6,3 \\
\hline \multicolumn{3}{|l|}{$\begin{array}{l}\text { Tratamiento de } \\
\text { neoplasia** }\end{array}$} \\
\hline Radioterapia & 8 & 25,8 \\
\hline Quimioterapia & 11 & 34,4 \\
\hline Hormonoterapia & 5 & 15,6 \\
\hline Otros & 8 & 25,8 \\
\hline
\end{tabular}

* Datos obtenidos de la Historia clínica.

** Se consideró el tratamiento recibido en la(s) neoplasia(s) previa(s).

La edad promedio al diagnóstico del primer cáncer primario fue de 66 años y un rango de 37 a 87 años, siendo esta edad menor en mujeres que en varones. La edad promedio al diagnóstico del segundo cáncer primario fue de 69 años y un rango de 41 a 94 años, que al igual que en el primer cáncer primario fue menor en mujeres (Tabla $\mathrm{N}^{\circ} 2$ ). Dos de los tres pacientes que presentaron cuatro cánceres primarios tuvieron una edad de presentación menor de la edad promedio de nuestro estudio (52 y 53 años). El primer cáncer primario más frecuente fue de mama y el segundo cáncer primario más frecuente fue de piel. (Tabla 3). La frecuencia absoluta de cáncer primario fue: primero (32), segundo (32), tercero (4) y cuarto (1).

\begin{tabular}{lllll} 
Sexo & Primer & Segundo & Tercer & Cuarto \\
Mujeres & 60 & 65 & 69 & 68 \\
Varones & 72 & 74 & 75 & 63 \\
En ambos sexos & 66 & 69 & 72 & 66 \\
\hline
\end{tabular}

Tabla 2. Frecuencia de los múltiples cánceres primarios y sitio anatómico en un hospital de Chiclayo, Perú durante los años 2009-2015.

\begin{tabular}{llc} 
Sitio anatómico & $\mathrm{N}$ & $\%$ \\
Piel & 17 & 53,1 \\
Próstata & 12 & 37,5 \\
Mama & 8 & 25,0 \\
Colon & 8 & 25,0 \\
Tiroides & 4 & 12,5 \\
Estómago & 3 & 9,4 \\
Útero & 3 & 9,4 \\
Ovario & 3 & 9,4 \\
Riñón & 3 & 9,4 \\
Sistema linfático & 3 & 9,4 \\
Intestino delgado & 2 & 6,3 \\
Meninges & 2 & 6,3 \\
Partes blandas & 2 & 6,3 \\
Orofaringe & 1 & 3,1 \\
\hline \hline
\end{tabular}

La asociación más frecuente encontrada fue piel-próstata, seguida de piel-colon. Se presentaron 31 cánceres metacrónicos, 14 de ellos en mujeres y 17 en varones. Los cánceres sincrónicos fueron 6 , presentándose todos en mujeres (Figura 1).

\section{Frecuencia de tipos de MCP según sexo}

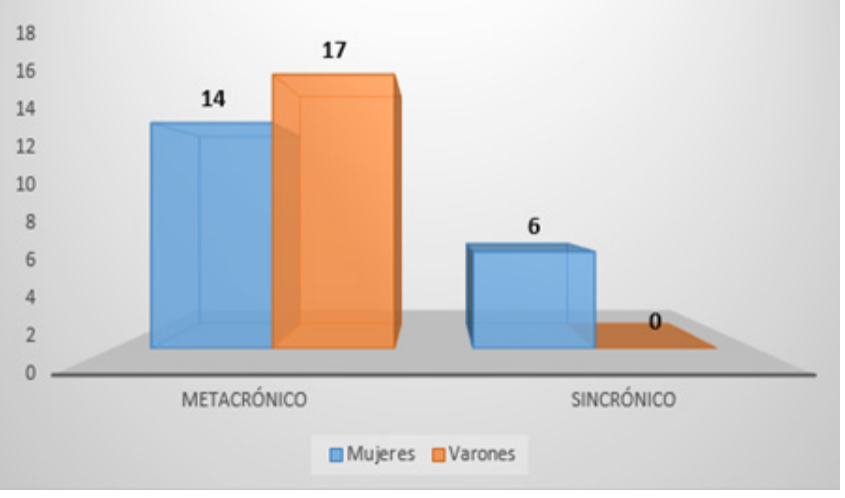

Figura 1: Frecuencia de tipos de cánceres primarios según sexo en un hospital de Chiclayo, Perú durante los años 2009-2015.

El grado de diferenciación más frecuente fue "desconocido", seguido de "medianamente diferenciado" (Tabla 4). 
Tabla $N^{\circ}$ 4: Frecuencia múltiples cánceres primarios según grado de diferenciación en un hospital de Chiclayo, Perú durante los años 20092015.

\begin{tabular}{lccccc} 
Grado de diferenciación & Primer & Segundo & Tercer & Cuarto & Total \\
$\begin{array}{l}\text { Bien diferenciado } \\
\begin{array}{l}\text { Medianamente diferen- } \\
\text { ciado }\end{array}\end{array}$ & 3 & 4 & 0 & 0 & 7 \\
$\begin{array}{l}\text { Pobremente diferen- } \\
\text { ciado }\end{array}$ & 5 & 4 & 1 & 1 & 11 \\
$\begin{array}{l}\text { Indiferenciado } \\
\text { Desconocido }\end{array}$ & 3 & 0 & 0 & 0 & 3 \\
\hline \hline
\end{tabular}

El intervalo promedio entre la admisión al hospital y el diagnóstico fue de 140 días para el primer cáncer primario y 181 días para el segundo cáncer primario (Tabla $\mathrm{N}^{\circ} 5$ ).

Tabla $\mathbf{N}^{\circ}$ 5: Intervalo de tiempo (días) entre la admisión al hospital y el diagnóstico para cada cáncer primario en un hospital de Chiclayo, Perú durante los años 2009-2015.

\begin{tabular}{lcccc} 
Grado de diferenciación & Primer & Segundo & Tercer & Cuarto \\
Mayor intervalo & 514 & 878 & 230 & 212 \\
Menor intervalo & 5 & 1 & 3 & - \\
Promedio & 140 & 181 & 117 & 212 \\
\hline \hline
\end{tabular}

La mayoría de los cánceres presentaron remisión, continuando algunos en control (Tabla $N^{\circ} 6$ ). Llamamos remisión a la ausencia clínica y/o ausencia de marcadores positivos para la enfermedad. Los pacientes que aún seguían en control tenían aún exámenes con resultados patológicos, pero con descenso en sus valores.

Tabla $\mathbf{N}^{\circ}$ 6: Número de cánceres que presentaron remisión en un hospital de Chiclayo, Perú durante los años 2009-2015.

\begin{tabular}{lccccc} 
Remisión & Primer & Segundo & Tercer & Cuarto & Total \\
Sí & 23 & 16 & 2 & 0 & 41 \\
No & 7 & 15 & 2 & 2 & 26 \\
Aún en controles & 2 & 1 & 0 & 1 & 4 \\
\hline \hline
\end{tabular}

\section{DISCUSIÓN}

En un estudio en China se encontró que el tipo de múltiples cánceres primarios (MCP) más frecuente fue metacrónico. Los tres sitios más frecuentes de cáncer fueron: cabeza y cuello, colorrectal y mama. La media del intervalo entre la primera y la segunda neoplasia fue de 5,5 años. La supervivencia media fue de 15,7 años ${ }^{(8)}$. Se halló en un estudio en Turquía en el que de 130 pacientes con MCP, 24 (18,4\%) presentaban neoplasia sincrónica y $106(81,6 \%)$ neoplasias metacrónicas, la media del intervalo entre la primera y la segunda neoplasia fue de 4,65 años para cáncer metacrónico y las asociaciones más frecuentes fueron mama-mama, mama-endometrio y mama-ovario ${ }^{(15)}$. En un análisis de 25 casos en Uruguay se encontró que, de 1884 pacientes, 25 $(1,3 \%)$ pacientes presentaban MCP, donde la edad media de presentación fue de 65 años, de los cuales 17 pacientes presentaron neoplasias metacrónicas, siendo la mediana de aparición entre el primer y segundo tumor de 11 meses y los sitios más frecuentes fueron: mama, piel, colon y riñón ${ }^{(10)}$.

Además, en estudios acerca de la supervivencia, los pacientes con neoplasias metacrónicas presentaban mayor sobrevida que los que poseían neoplasias sincrónicas ${ }^{(8)}$. Basándonos en el aumento de frecuencia y el impacto de las neoplasias en la calidad de vida y costos económicos de quienes las padecen es que se decidió realizar este estudio, además de que los estudios previos fueron realizados en otros países, cuya realidad difiere de la nuestra (16). Además, orientará a los profesionales de salud a continuar con el seguimiento de los pacientes que ya padecen de uno, incluso si ha remitido, por el posible hallazgo de un segundo tumor primario.

En la última década se ha mostrado el incremento en la frecuencia de MCP. En nuestro estudio se encontró que la frecuencia de MCP fue de 1,1\%, esta misma frecuencia se encontró en un estudio realizado por Demandante et al, 2003 donde revisaron la frecuencia de MCP de estudios publicados desde el año 1966 al 2000 encontrando una frecuencia que varía de $0,73-11,7 \%{ }^{(9)}$. Así mismo se observó una frecuencia de $1,1 \%$ en un estudio retrospectivo con 72 pacientes en China ${ }^{(8)}$. Los artículos publicados reportan una tendencia variada con respecto al sexo más frecuente en las MCP. En nuestro estudio se encontró que fue más frecuente en mujeres que en varones, siendo esta diferencia no muy marcada, 15 y 17 casos, respectivamente.

En nuestro estudio el primer cáncer primario más frecuente fue de mama mientras que otros estudios publicados reportan como cáncer primario más frecuente los de laringe, vejiga y pecho. Como segundo cáncer primario, el más frecuente fue piel, mientras que en otros estudios fueron pulmón y colon (15,17). El tipo de cáncer más frecuente fue el cáncer metacrónico, tanto en varones como en mujeres, lo cual concuerda con las publicaciones internacionales sobre múltiples cánceres primarios ${ }^{(8,16,19)}$.

La edad de presentación fue menor para los pacientes con cáncer sincrónico siendo este resultado controversial con las demás publicaciones, en los cuales los cánceres metacrónicos son los de menor edad de presentación ${ }^{(8,15)}$. La definición de cánceres sincrónicos y metacrónicos hace referencia al momento del diagnóstico, tomando como punto de corte 6 meses del siguiente tumor primario, lo cual implicaría que un paciente puede padecer de cáncer sincrónico, pero si no se diagnostica a tiempo puede ser catalogado como cáncer metacrónico. Esta situación podría tornarse relevante en nuestro medio en donde el 
tiempo de diagnóstico demora de manera importante debido a diversas situaciones, pudiendo así ser este el motivo de mayor frecuencia de cánceres metacrónicos, sin embargo, este hallazgo coincide con lo reportado en la literatura internacional.

Continuando con lo anteriormente mencionado, se encontró que el intervalo promedio entre la admisión al hospital y el diagnóstico de cáncer fue de 140 días para el primer cáncer primario y de 181 días para el segundo cáncer primario. Estos intervalos prolongados no son más que el reflejo de las condiciones actuales de atención de salud en nuestro país, lo cual influye en el diagnóstico del tipo de cáncer encontrado (metacrónico o sincrónico) y en el tiempo posible de haber presentado los cánceres sin tratamiento y de la progresión de este.

El grado de diferenciación (etapa clínica) más frecuente fue el "medianamente diferenciado" (Grado II), lo cual manifiesta el estado de los pacientes a la hora de buscar atención médica el cual se encuentra influenciado por la cultura de la población ${ }^{(18)}$, a esto se le agrega lo mencionado anteriormente acerca de las condiciones de salud en el rápido diagnóstico desde la admisión del paciente al hospital. Dentro de los factores de riesgo encontrados, el tratamiento dado para la neoplasia previa fue el más frecuente. Encabezando lo mencionado estuvo la quimioterapia seguida de la radioterapia (porcentajes). Así mismo, se ha encontrado que muchos de los pacientes en diferentes estudios recibieron quimioterapia, radioterapia, hormonoterapia y combinación radio/quimioterapia dentro de lo que se menciona que son factores influyentes en el desarrollo de los MCP, puesto que se ha visto en un gran porcentaje como carcinogénicos ${ }^{(12,13,19)}$.

Se mencionan, también, otros carcinógenos, aparte de quimioterapia y radioterapia, como el tabaco, alcohol y susceptibilidad genética como posibles factores que elevarían el riesgo de MCP. El tabaquismo y alcoholismo son factores de riesgo para el desarrollo de cáncer. En nuestro estudio hemos considerado "Consumo de tabaco" y "Consumo de alcohol" debido a que en las historias clínicas solo se plasma el consumo o no de estos hábitos nocivos, mas no su cantidad y frecuencia. En nuestro estudio debido a la limitación de no tener el dato bien elaborado, ya que en las historias clínicas solo se consignaba "Fumaba o no" y si "Ingería bebidas alcohólicas o no", no se puede corroborar este hallazgo. Se considera pertinente que con los resultados encontrados en el presente estudio y en los demás estudios revisados, los médicos, en especial los oncólogos, deben tener presente que los pacientes con un primer cáncer primario presentan un mayor riesgo de desarrollar otro cáncer primario, por lo que no se debe descuidar su seguimiento incluso habiendo encontrado remisión. Así mismo, educar al paciente e informarle la posibilidad que existe de que aparezca una nueva neoplasia primaria y estar atentos en la presencia de nuevos signos y/o síntomas que podrían indicar la presencia de un nuevo cáncer primario. Esto incluye pacientes con 2 o más cánceres primarios, puesto que se ha visto que incluso remitiendo 2 cánceres primarios pueden aparecer un tercero y/o un cuarto cáncer primario.

Una de las limitaciones del estudio fue el tamaño de muestra reducido, esto se dio por el mal llenado de la historia clínica y la falta de datos. Del total de pacientes de MCP que fueron 89 , solo 32 se consideraron en nuestro estudio debido a la falta de accesibilidad de las historias clínicas por una inadecuada organización de estas por parte del hospital donde se realizó el estudio, además las historias clínicas de los pacientes fallecidos no se tuvo accesibilidad. Para futuros estudios se debe mejorar el registro de las historias clínicas, así como su completo llenado para lograr una adecuada recolección de información. Se considera pertinente que se realicen estudios con más población e incluir pacientes fallecidos y no fallecidos como parte de la población de estudio.

Concluimos los múltiples cánceres primarios fueron más frecuentes en el sexo femenino. Los factores de riesgo son el tratamiento de la neoplasia previa. La edad promedio al diagnóstico del primer cáncer primario fue menor que en varones. El primer cáncer primario más frecuente fue de mama. La asociación más frecuente fue piel-próstata. El tipo de cáncer más frecuente fue el cáncer metacrónico tanto en varones como en mujeres. Ningún varón presentó cáncer sincrónico.

Conflictos de interés: declaramos no tener conflictos de interés en esta publicación.

Fuentes de financiamiento: autofinanciado.

\section{REFERENCIAS BIBLIOGRÁFICAS}

1. Ramos W, Venegas D, Medina J, Guerrero C, Cruz A. Análisis de la Situación del Cáncer en el Perú, 2013. Perú: MINSA; 2013.

2. Organización Panamericana de la Salud. Cáncer [Sede web]. USA:OPS;2012

3. Billroth T. Die allgemeine chirurgische Pathologie und Therapie: in fünfzig Vorlesungen : ein Handbuch für Studirende und Aerzte. G. Reimer; 1863. $742 \mathrm{p}$.

4. Warren S, Puertas O. Múltiples tumores malignos primarios: encuesta de la literatura y de un estudio estadístico. Am J Cáncer. 1932; $16: 1358-414$.

5. Chong V, Idros A, Telisinghe P. Triple synchronous gastrointestinal malignancies: a rare occurrence. Singapore Med J. 2010; 51(10):e177.

6. Jiao F, Hu H, Wang L-W. Quadruple primary malignancy patient with survival time more than 20 years. World J Gastroenterol WJG. 2013; 19(9):1498-501.

7. IACR Conference 2013 Buenos Aires, Argentina [Sede web]. 2015 [citado 18 de abril de 2015]. Disponible en: http://www.iacr2013.com.

8. Jiao F, Yao L-J, Zhou J, Hu H, Wang L-W. Clinical features of multiple primary malignancies: a retrospective analysis of 72 Chinese patients. Asian Pac J Cancer Prev APJCP. 2014;15(1):331-4

9. Demandante C, Troyer D, Miles T. Multiple primary malignant neoplas- 
ms: case report and a comprehensive review of the literature. Am J Clin Oncol. 2003; 26(1):79-83.

10. Santander G, Lombardo K, Rodríguez R. Tumores múltiples Análisis de 25 casos. Sanidad Militar. 2006; 28(1):73-80.

11. Pastore A, Palleschi G, Autieri D, Leto A, Ripoli A, Maggioni C, et al Synchronous primary neoplasms of the bladder, skin and breast in a male patient: a case report. World J Surg Oncol. 2013; 11:282.

12. Koutsopoulos A, Dambaki K, Datseris G, Giannikaki E, Froudarakis $\mathrm{M}$, Stathopoulos $\mathrm{E}$. A novel combination of multiple primary carcinomas: Urinary bladder transitional cell carcinoma, prostate adenocarcinoma and small cell lung carcinoma- report of a case and review of the literature. World J Surg Oncol. 2005; 3:51.

13. Yamamoto D, Hamada $Y$, Tsubota $Y$, Kawakami K, Yamamoto C, Yamamoto M. Simultaneous development of adenocarcinoma and gastrointestinal stromal tumor (GIST) in the stomach: case report. World J Surg Oncol. 2012; 10:6.

14. Limaylla $\mathrm{H}$, Alfaro $\mathrm{A}$, León $\mathrm{M}$. Tumor sincrónico: cáncer de colon y liposarcoma. Anales de la Facultad de Medicina. 2013; 74(2): 149-52.

15. Arpaci E, Tokluoglu S, Yetigyigit T, Necati A. Multiple Primary Malignancies- A Retrospective Analysis at a Single Center in Turkey. APJCP. 2013; 14(2): 769-772

16. Muela A, Jorquera F, Malagón R, Ribas M, Muñoz M. Neoplasias malignas múltiples en pacientes con cáncer de próstata en el área sanitaria de León. An Med Interna.2005; 22(1):9-14.

17. Gursel B, Meydan D, Özbek N,Ozdemir O, Odabas E. Multiple Primary Malignant Neoplasms from the black Sea Region of Turkey. AP JCP. 2011; 39: 667-674.

18. Villegas C, Chancón A, Cardona J, Correa L. Perfil clínico epidemiológico de los pacientes con cánceres tratados en una institución de tercer nivel. Manizales, Colombia, 1995 - 2004. Colomb Med. 2012; 43: 11-18.

19. Chirila D, Turdeanu N, Constantea N, Coman I, Pop T, Popp R, Blacescu O, Vesa S. Multiple Malignant Tumors. Chirurgia. 2013; 4: 498 $-502$ 